\title{
A general approach to the analysis and description of partially polarized light in rigorous grating theory
}

Jani Tervo

jani.tervo@joensuu.fi

Ismo A. Turunen

Benfeng Bai

\author{
Department of Physics and Mathematics, University of Joensuu, P.O. Box 111, FI-80101 Joensuu, Fin- \\ land \\ Department of Physics and Mathematics, University of Joensuu, P.O. Box 111, FI-80101 Joensuu, Fin- \\ land \\ Department of Physics and Mathematics, University of Joensuu, P.O. Box 111, FI-80101 Joensuu, Fin- \\ land
}

Simple and intuitive principle is put forward to analyze the grating diffraction problem and to describe the polarization properties of diffraction orders. The method is applicable for any state of polarization of the input plane wave, including partially polarized or unpolarized fields. [DOI: $10.2971 /$ jeos.2008.08004]

Keywords: Diffraction gratings, grating theory, polarization

\section{INTRODUCTION}

The electromagnetic theory of gratings [1] has undergone many remarkable changes during the last decades. In particular, rapid development of computers has made possible to analyze rigorously, not only simple linear gratings, but also twodimensionally periodic gratings, also called crossed gratings (see, e.g. [2]-[6]). Since the main focus of the grating theory has been in the development of numerical efficiency, the properties of the input and output fields, except for the diffraction efficiencies, have not received much attention, even though they are of course in the central role in practical applications. For example, the state of polarization of the input field is almost exclusively assumed to be linear, although the theory internally assumes general, elliptically polarized input.

The situation becomes more complex if the input field is not anymore fully polarized, but either unpolarized or partially polarized, which is becoming more and more common situation, since, for example, LED illumination is employed in combination with grating theory, e.g., in display applications [7]-[9]. The unpolarized case is still quite manageable even without careful considerations, as one may very well take the sum the intensities of the uncorrelated polarization components after solving the grating-diffraction problem. However, the diffraction orders are, in general, not anymore unpolarized, but more or less partially polarized. Thus, if the diffraction orders are desired to be used as inputs to another optical elements, their exact polarization properties are of interest. An exemplar of such a system is the double-sided grating analyzed in [10].

In this article, we put forward a simple and intuitive way to perform the grating analysis for input illumination with any state of polarization. The method is based on the well-known representation of polarization in terms of Poincare's sphere and the degree of polarization, and works with practically any rigorous method. However, in the derivation we employ the $S$-matrix (scattering matrix) approach [11] which is assumed to be known by the reader.

\section{DIFFRACTION GEOMETRY}

Assume a diffracting structure which is periodically modulated in one or two directions that are perpendicular to the $z$-axis, i.e. the normal vector of the structure is parallel to the $z$ axis. Without a loss of generality, we may assume that the first (or only) direction of modulation is the $x$ direction. We also assume, for simplicity, that the input field is a plane wave, with its propagation direction defined by the wave vector $\mathbf{k}_{\mathrm{in}}$, such that $k_{\mathrm{in}, z}>0$, and the complex amplitude of the electric field is denoted by $\mathbf{e}_{\text {in }}$, where the subscript in denotes the input field. Note that in the following analysis, we employ the spatial frequency-temporal frequency representation of the field quantities, i.e. we examine one temporal frequency at a time. Thus, the properties of $\mathbf{e}_{\text {in }}$ are frequency-dependent but we omit the explicit dependence because of brevity. In general, $\mathbf{e}_{\text {in }}$ is a random vector, i.e. both its magnitude and direction are known only in statistical terms, which permits us to analyze, not only deterministic fields, but also partially polarized light.

As usual, we analyze the properties of the input field with the help of the plane of incidence, i.e. the plane defined by the wave vector and the surface normal of the grating. The angle between the wave vector and the $z$ axis is denoted by $\theta_{\text {in, }}$ and the angle between the input plane and the $x$ axis is denoted by $\phi_{\text {in }}$ (see Figure 1 for definitions). Thus, the components of 


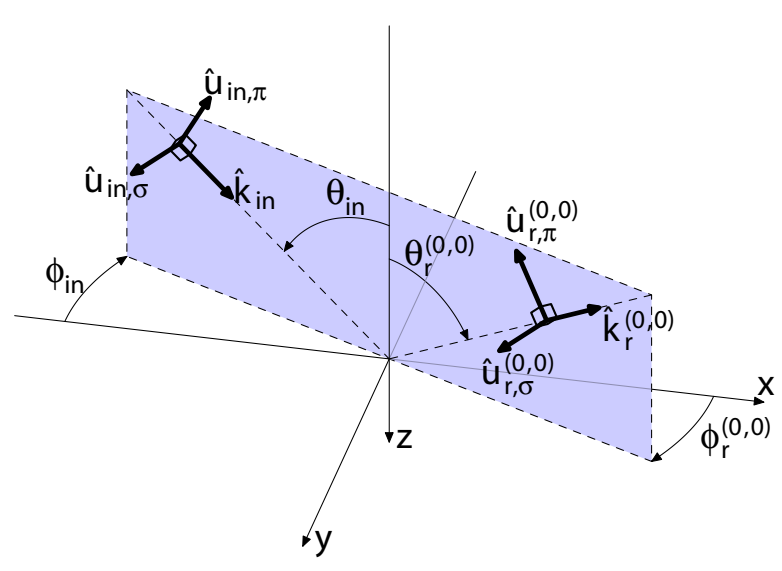

FIG. 1 Basic definitions of the geometry. Only the zeroth reflected order is shown for clarity.

the input wave vector are related to the angles as follows:

$$
\begin{aligned}
& k_{\mathrm{in}, x}=k_{\mathrm{in}} \sin \theta_{\mathrm{in}} \cos \phi_{\mathrm{in}} \\
& k_{\mathrm{in}, y}=k_{\mathrm{in}} \sin \theta_{\mathrm{in}} \sin \phi_{\mathrm{in}}, \\
& k_{\mathrm{in}, z}=k_{\mathrm{in}} \cos \theta_{\mathrm{in}},
\end{aligned}
$$

The electric-field components of the input plane wave are best described in the $\pi-\sigma$ basis (also known as p-s and TM-TE bases), in which the unit vectors $\hat{\mathbf{u}}_{\mathrm{in}, \pi}$ and $\hat{\mathbf{u}}_{\mathrm{in}, \sigma}$ are parallel and perpendicular, respectively, to the input plane:

$$
\begin{aligned}
& e_{\mathrm{in}, \pi}=e_{\mathrm{in}, x} \cos \theta_{\mathrm{in}} \cos \phi_{\mathrm{in}}+e_{\mathrm{in}, y} \cos \theta_{\mathrm{in}} \sin \phi_{\mathrm{in}}-e_{\mathrm{in}, z} \sin \theta_{\mathrm{in}} \\
& e_{\mathrm{in}, \sigma}=-e_{\mathrm{in}, x} \sin \phi_{\mathrm{in}}+e_{\mathrm{in}, y} \cos \phi_{\mathrm{in} .}
\end{aligned}
$$

If $\mathbf{k}$ is parallel to the $z$ axis, in which case the choice of the field directions is not unique, we may very well fix $\hat{\mathbf{u}}_{\mathrm{in}, \pi}$ parallel to the $x$ axis.

The propagating diffraction orders are analyzed analogously: For each transmitted and reflected diffraction order $(m, n)$, we define the angles $\theta_{j}^{(m, n)}$ and $\phi_{j}^{(m, n)}$ as well as the field components $e_{j, \pi}^{(m, n)}$ and $e_{j, \sigma}^{(m, n)}$, where $j=\mathrm{t}$ for transmitted waves and and $j=\mathrm{r}$ for reflected waves. Here the angles and components are defined with respect to the so-called exit plane of the diffraction order, defined by the wave vector $\mathbf{k}_{j}^{(m, n)}$ of the order and the $z$ axis. The definitions of the wave-vector components are analogous to Eqs. (1a)-(1b), except that $k_{\mathrm{r}, z}^{(m, n)}=$ $-k_{\mathrm{in}} \cos \theta_{\mathrm{r}}^{(m, n)}$. Also definitions for $e_{\mathrm{t}, \pi}^{(m, n)}, e_{\mathrm{t}, \sigma}^{(m, n)}$, and $e_{\mathrm{r}}, \sigma^{(m, n)}$ are completely analogous to Eqs. (2a) and (2b), but the $\pi$ components of the reflected orders are obtained from

$$
\begin{aligned}
e_{\mathrm{r}, \pi}^{(m, n)}= & -e_{\mathrm{r}, x}^{(m, n)} \cos \theta_{\mathrm{r}}^{(m, n)} \cos \phi_{\mathrm{r}}^{(m, n)} \\
& -e_{\mathrm{r}, y}^{(m, n)} \cos \theta_{\mathrm{r}}^{(m, n)} \sin \phi_{\mathrm{in}}^{(m, n)}-e_{\mathrm{r}, z}^{(m, n)} \sin \theta_{\mathrm{r}}^{(m, n)} .
\end{aligned}
$$

Our definitions are thus analogous to the ones used, e.g., in [12]: the wave vector, $\pi$-unit vector and $\sigma$-unit vector form a right-handed triplet for every order. Note that the abovegiven definitions hold only for propagating diffraction orders: The polarization analysis of evanescent fields does not usually involve the analysis of different spatial frequencies.

\section{GENERAL CONCEPTS OF PARTIALLY POLARIZED AND PARTIALLY COHERENT LIGHT}

Let us next recall some well known basic concepts of partially polarized planar wave fields. The second-order statistical properties of a planar wave field in the space-frequency are usually described by a spectral polarization matrix (also known as a coherency matrix) $[13,14]$

$$
\mathbf{J}=\left\langle\mathbf{e}^{*} \mathbf{e}^{\mathrm{T}}\right\rangle=\left[\begin{array}{ll}
\left\langle\left|e_{\pi}\right|^{2}\right\rangle & \left\langle e_{\pi}^{*} e_{\sigma}\right\rangle \\
\left\langle e_{\sigma}^{*} e_{\pi}\right\rangle & \left\langle\left|e_{\sigma}\right|^{2}\right\rangle
\end{array}\right]=\left[\begin{array}{cc}
J_{\pi \pi} & J_{\pi \sigma} \\
J_{\sigma \pi} & J_{\sigma \sigma}
\end{array}\right],
$$

where $\mathbf{e}=\left[e_{\pi}, e_{\sigma}\right]^{\mathrm{T}}$ denotes the realization of a (random) electric field. Here the angle brackets denote the average over the statistical ensemble of the possible field realizations, the asterisk denotes complex conjugation, and T denotes the transpose operation. It is obvious from Eq. (3) that $\mathbf{J}$ is Hermitian, i.e. $\mathbf{J}^{\dagger}=\mathbf{J}$, where the dagger denotes the adjoint matrix. Moreover, $\mathbf{J}$ is also non-negative definite, i.e. for any vector $\mathbf{v}$, we have $\mathbf{v}^{\dagger} \mathbf{J v} \geq 0$. In the diffraction problem discussed in the preceding Section, we may employ the definition (3) in the analysis of either the input plane wave or any of the propagating diffraction orders. For those readers who are not familiar with the polarization matrix, we point out that each element of $\mathbf{J}$ is measurable using simple optical elements (see, e.g. Ref. [13]). In particular, the trace of the matrix is proportional to the intensity of the field.

Another commonly employed description of partially polarized radiation is the Stokes representation, in which one uses the four Stokes parameters $[13,14]$, denoted here by $S_{q}, q=$ $0 \ldots 3$, that are related to the elements of the polarization matrix as follows:

$$
\begin{array}{ll}
S_{0}=J_{\pi \pi}+J_{\sigma \sigma}, & S_{1}=J_{\pi \pi}-J_{\sigma \sigma}, \\
S_{2}=2 \Re\left(J_{\pi \sigma}\right), & S_{3}=2 \Im\left(J_{\pi \sigma}\right),
\end{array}
$$

where $\Re$ and $\Im$ denote the real part and the imaginary part, respectively. It is also customary to define the normalized Stokes parameters $s_{q}=S_{q} / S_{0}, q=1 \ldots 3$. In the Stokes notation, the degree of polarization takes on the form

$$
P=\left(s_{1}^{2}+s_{2}^{2}+s_{3}^{2}\right)^{1 / 2},
$$

whereas the diffraction efficiency of the order $(m, n)$ is

$$
\eta_{j}^{(m, n)}=\frac{\cos \theta_{j}^{(m, n)} S_{0, j}^{(m, n)}}{\cos \theta_{\mathrm{in}} S_{0, \mathrm{in}}}, \quad j=(\mathrm{t}, \mathrm{r}) .
$$

where we have clearly assumed that only propagating orders are considered.

The strength of Stokes representation is that the three normalized Stokes parameters $s_{q}$ can be employed to graphically illustrate the state of polarization in Poincare's sphere [14]. Namely, by assuming three orthogonal directions, denoted by unit vectors $\hat{\mathbf{s}}_{q}, q=1 \ldots 3$, and defining the Poincare vector [14] $\mathbf{p}=s_{1} \hat{\mathbf{s}}_{1}+s_{2} \hat{\mathbf{s}}_{2}+s_{3} \hat{\mathbf{s}}_{3}$, each state of polarization is uniquely related to one point in a unit sphere: The direction of the vector, characterized by the spherical-polar-coordinate angles $\vartheta$ and $\varphi$, related to the Stokes parameters by

$$
s_{1}=P \sin \vartheta \cos \varphi, \quad s_{2}=P \sin \vartheta \sin \varphi, \quad s_{3}=P \cos \vartheta,
$$


gives us the type of polarization, and the length of the vector, in view of Eq. (5), gives the degree of polarization. For graphical illustration of the geometry, see Figure 2.

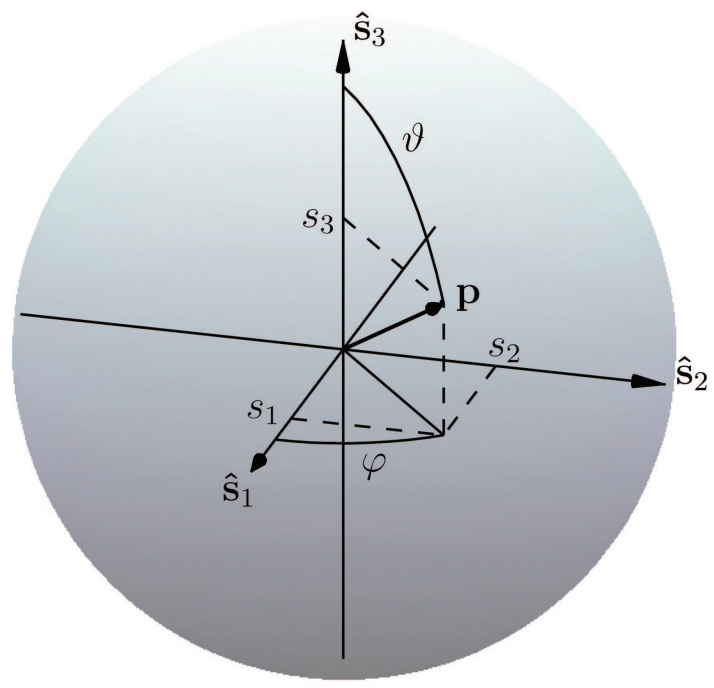

FIG. 2 Poincare's sphere and the definition of angles $\vartheta$ and $\varphi$.

The parameters $(\eta, \theta, \phi, P, \vartheta, \varphi)$ thus provide us the convenient knowledge of the properties of certain plane-wave component. The first three parameters give us the standard knowledge on diffraction efficiency and propagation direction, whereas the last three parameters give us full knowledge on the polarization properties of the wave, including partial polarization. However, since the phase is, in general, a random quantity, the phase information can be only given through the correlation properties between the orders. To this end, one must introduce either the cross-spectral density matrix $[13,15]$ or the angular correlation matrix $[13,16]$, the latter of which is particularly useful in the diffraction-order analysis. Since we have assumed that the input field is a plane wave, the angular correlation matrix is of a discrete form:

$$
\begin{aligned}
\mathbf{A}_{j ; l}^{(m, n ; p, q)} & =\left\langle\mathbf{e}_{j}^{(m, n)^{*}} \mathbf{e}_{l}^{(p, q)^{\mathrm{T}}}\right\rangle \\
& =\left[\begin{array}{ll}
\left\langle e_{j, \pi}^{(m, n)^{*}} e_{l, \pi}^{(p, q)}\right\rangle & \left\langle e_{j, \pi}^{(m, n)^{*}} e_{l, \sigma}^{(p, q)}\right\rangle \\
\left\langle e_{j, \sigma}^{(m, n)^{*}} e_{l, \pi}^{(p, q)}\right\rangle & \left\langle e_{j, \sigma}^{(m, n)^{*}} e_{l, \sigma}^{(p, q)}\right\rangle
\end{array}\right],
\end{aligned}
$$

where $j=(\mathrm{t}, \mathrm{r})$ and $l=(\mathrm{t}, \mathrm{r})$. Thus, the elements of the angular correlation matrix describe the cross-correlation between any two components of any two diffraction orders. This matrix thus contains the information on the statistical correlation between the components of two diffraction orders. Note that if the orders are the same, i.e. $j=l, m=p$, and $n=q$, the angular correlation matrix coincides with the polarization matrix of the examined order. Analogously, we can also define the angular correlation matrix between the input-field components and one of the diffraction orders, in which case we employ the notations

$$
\begin{aligned}
& \mathbf{A}_{\mathrm{in} ; j}^{(m, n)}=\left\langle\mathbf{e}_{\mathrm{in}}^{*} \mathbf{e}_{j}^{(m, n)^{\mathrm{T}}}\right\rangle, \\
& \mathbf{A}_{j ; \mathrm{in}}^{(m, n)}=\left\langle\mathbf{e}_{j}^{(m, n)^{*}} \mathbf{e}_{\mathrm{in}}^{\mathrm{T}}\right\rangle, \quad j=(\mathbf{t}, \mathbf{r}) .
\end{aligned}
$$

Note that if there is full correlation between the input-field components, i.e. if $\left|J_{\text {in }, \pi \sigma}\right|^{2}=J_{\text {in, } \pi \pi} J_{\text {in }, \sigma \sigma}$, which corresponds to the case traditionally assumed in the grating analysis, the arguments of the diagonal elements of the angular correlation functions of diffraction orders correspond to the phase information.

The definitions presented above provide us with the full information of the field within the framework of the second-order statistical theory of light. In the next Section, we shall study how this information can be efficiently employed in rigorous diffraction theory of gratings.

\section{RIGOROUS GRATING THEORY WITH PARTIALLY POLARIZED INPUT FIELD}

Assume next that we know the parameters $\left(\theta_{\text {in }}, \phi_{\text {in }}, P_{\text {in }}, \vartheta_{\text {in }}, \varphi_{\text {in }}\right)$ for the input plane wave, and we desire to find out the same parameters for every diffraction order, as well as their diffraction efficiencies. In the most typical case encountered in the rigorous diffraction theory of gratings, the field must be input to the grating code as two electric-field components. In basic Fourier Modal Method (FMM) $[2,4]$ these components are $x$ and $y$ components of the electric field, but they can be also other components. For each order, the transformations between the $\pi-\sigma$-basis and the grating coordinate basis can be represented by matrices $\mathbf{T}_{j, \pi \sigma \rightarrow \mathrm{g}}^{(m, n)}$ and $\mathbf{T}_{j, \mathrm{~g} \rightarrow \pi \sigma^{\prime}}^{(m, n)} j=(\mathrm{r}, \mathrm{t})$. Analogous matrices can be naturally defined also for the input field, in which case we use notations $\mathbf{T}_{\mathrm{in}, \pi \sigma \rightarrow \mathrm{g}}$ and $\mathbf{T}_{\mathrm{in}, \mathrm{g} \rightarrow \pi \sigma}$.

In order to employ the $S$-matrix approach [11], let us arrange the complex amplitudes of transmitted and reflected diffraction orders into column vectors $\mathbf{E}_{\mathrm{t}, \mathrm{g}}$ and $\mathbf{E}_{\mathrm{r}, \mathrm{g}}$, respectively. Note that the ordering of the elements in the vectors in the $S$ matrix algorithm is free. Also the complex amplitudes of the input plane wave are arranged in a column vector $\mathbf{E}_{\mathrm{in}, \mathrm{g}}$ that usually contains mostly zeros (other plane-wave components in the Rayleigh basis). For example, in basic FMM only two of the elements are non-zero. The $S$-matrix equation now reads

$$
\left[\begin{array}{l}
\mathbf{E}_{\mathrm{t}, \mathrm{g}} \\
\mathbf{E}_{\mathrm{r}, \mathrm{g}}
\end{array}\right]=\mathbf{S}\left[\begin{array}{c}
\mathbf{E}_{\mathrm{in}, \mathrm{g}} \\
\mathbf{0}
\end{array}\right]
$$

where $\mathbf{S}$ is the system $S$-matrix and $\mathbf{0}$ is a zero-vector of the same size as $\mathbf{E}_{\mathrm{in}, \mathrm{g}}$. Note that here we have assumed that the grating is illuminated from one side only, which is almost exclusively the case in practice.

It is obvious from Eq. (10) that, for every diffraction order, we have a sub-matrix $\mathbf{S}_{j}^{(m, n)}$ of $\mathbf{S}$ for which

$$
\mathbf{e}_{j, \mathrm{~g}}^{(m, n)}=\mathbf{S}_{j}^{(m, n)} \mathbf{e}_{\mathrm{in}, \mathrm{g}}, \quad j=(\mathrm{t}, \mathrm{r}) .
$$

On the other hand, since $\mathbf{e}_{j, \pi \sigma}^{(m, n)}=\mathbf{T}_{j, \mathrm{~g} \rightarrow \pi \sigma}^{(m, n)} \mathbf{e}_{j, \mathrm{~g}}^{(m, n)}$ and $\mathbf{e}_{\mathrm{in}, \mathrm{g}}=$ $\mathbf{T}_{\mathrm{in}, \pi \sigma \rightarrow \mathrm{g}} \mathbf{e}_{\mathrm{in}, \pi \sigma}$, we have at once

$$
\begin{aligned}
\mathbf{e}_{j, \pi \sigma}^{(m, n)} & =\mathbf{T}_{j, \mathrm{~g} \rightarrow \pi \sigma}^{(m, n)} \mathbf{S}_{j}^{(m, n)} \mathbf{T}_{\mathrm{in}, \pi \sigma \rightarrow \mathrm{g}} \mathbf{e}_{\mathrm{in}, \pi \sigma}, \\
& =\mathbf{M}_{j}^{(m, n)} \mathbf{e}_{\mathrm{in}, \pi \sigma}, \quad j=(\mathbf{t}, \mathbf{r}),
\end{aligned}
$$

where

$$
\mathbf{M}_{j}^{(m, n)}=\mathbf{T}_{j, \mathrm{~g} \rightarrow \pi \sigma}^{(m, n)} \mathbf{S}_{j}^{(m, n)} \mathbf{T}_{\mathrm{in}, \pi \sigma \rightarrow \mathrm{g}}, \quad j=(\mathrm{t}, \mathrm{r}),
$$


are $2 \times 2$ matrices that describe how the input electric field is diffracted to the reflected and transmitted orders.

Note that, for given input angles $\theta_{\text {in }}$ and $\phi_{\text {in }}, \mathbf{M}_{j}^{(m, n)}$ are fixed, i.e., they do not depend on the polarization properties of the input field. Therefore, even though the input electric-field vector $\mathbf{e}_{\mathrm{in}, ß œ \mathrm{e}}$ is generally a random one, the matrices $\mathbf{M}_{j}^{(m, n)}$ are fully deterministic. Thus, by inserting Eq. (13) into Eq. (3), we obtain the polarization matrices for reflected and transmitted orders:

$$
\mathbf{J}_{j, \pi \sigma}^{(m, n)}=\left[\mathbf{M}_{j}^{(m, n)}\right]^{*} \mathbf{J}_{\mathrm{in}, \pi \sigma}\left[\mathbf{M}_{j}^{(m, n)}\right]^{\mathrm{T}}, \quad j=(\mathbf{t}, \mathbf{r}) .
$$

These equations give us full and rigorous information about the polarization states of the diffraction orders, including partial polarization. However, it is possible to avoid solving the submatrices $\mathbf{M}_{j}^{(m, n)}$, as we shall see in the next Section.

\section{UTILIZATION OF THE EIGENDECOMPOSITION OF THE POLARIZATION MATRIX}

Let us recall from Eq. (3) that the polarization matrix is always both Hermitian and non-negative definite square matrix. It can be therefore decomposed into the form [17]

$$
\mathbf{J}=\mathbf{J}_{1}+\mathbf{J}_{2}
$$

where

$$
\mathbf{J}_{p}=I_{p} \mathbf{e}_{p}^{*} \mathbf{e}_{p}^{\mathrm{T}}, \quad p=(1,2) .
$$

Here $I_{p}$ and $\mathbf{e}_{p}^{*}, p=(1,2)$ are the eigenvalues and eigenvectors, respectively, of the polarization matrix. Moreover, the eigenvalues are real and non-negative, and the different eigenvectors are orthonormal, i.e. $\mathbf{e}_{p}^{\dagger} \mathbf{e}_{q}=\delta_{p, q}$, where $\delta_{p, q}$ is the Kronecker symbol. The polarization matrices $\mathbf{J}_{1}$ and $\mathbf{J}_{2}$ represent fully polarized, but mutually uncorrelated fields. The vectors $\mathbf{e}_{1}$ and $\mathbf{e}_{2}$ represent their polarization states, and $I_{1}$ and $I_{2}$ are proportional to their intensities.

We next consider the polarization matrix of the input field $\mathbf{J}_{\text {in }, \pi \sigma}$, with its eigenvectors and eigenvalues likewise denoted by a subscript in. Note that here the subscript $\pi \sigma$ denotes the $\pi-\sigma$ basis, rather than the off-diagonal element of the matrix. We first assume that the grating is illuminated only by a field with intensity $I_{\text {in, } 1}$ and polarization state $\mathbf{e}_{\mathrm{in}, 1, \pi \sigma}$. This clearly permits us to apply the standard $S$-matrix approach, yielding the output diffraction orders in terms of vectors $\mathbf{e}_{\mathrm{t}, 1, \mathrm{~g}}^{(m, n)}$ and $\mathbf{e}_{\mathrm{r}, 1, \mathrm{~g}}^{(m, n)}$. These can be converted at once into the $\pi-\sigma$-basis vectors $\mathbf{e}_{\mathrm{t}, 1, \pi \sigma}^{(m, n)}$ and $\mathbf{e}_{\mathrm{r}, 1, \pi \sigma}^{(m, n)}$. The polarization matrices of the orders are now of the form

$$
\mathbf{J}_{j, 1, \pi \sigma}^{(m, n)}=I_{\mathrm{in}, 1}\left[\mathbf{e}_{j, 1, \pi \sigma}^{(m, n)}\right]^{*}\left[\mathbf{e}_{j, 1, \pi \sigma}^{(m, n)}\right]^{\mathrm{T}}, \quad j=(\mathrm{t}, \mathrm{r}) .
$$

Analogously, if we assume illumination only by a field with intensity $I_{\mathrm{in}, 2}$ and polarization state $\mathbf{e}_{\mathrm{in}, 2, \pi \sigma}$, we obtain

$$
\mathbf{J}_{j, 2, \pi \sigma}^{(m, n)}=I_{\text {in, }, 2}\left[\mathbf{e}_{j, 2, \pi \sigma}^{(m, n)}\right]^{*}\left[\mathbf{e}_{j, 2, \pi \sigma}^{(m, n)}\right]^{\mathrm{T}}, \quad j=(\mathbf{t}, \mathbf{r}) .
$$

Employing Eqs. (12) and (14) we immediately find that

$$
\mathbf{J}_{j, \pi \sigma}^{(m, n)}=\mathbf{J}_{j, 1, \pi \sigma}^{(m, n)}+\mathbf{J}_{j, 2, \pi \sigma}^{(m, n)} .
$$

Thus, illumination by fields characterized by the eigenvectors are eigenvalues of the original polarization matrix yields exactly the correct polarization matrices of the output orders. An important consequence of this result is that one does not need to find out the submatrices $\mathbf{M}_{j}^{(m, n)}$ from the grating $S$-matrix. Instead, it is possible to use the existing grating code directly, assuming separate illuminations with fields characterized by eigenvalues and eigenvectors of the input-field polarization matrix.

It is obvious that the eigenvector-based approach is based on the linearity of the $S$-matrix approach and, on the other hand, on the fact that the eigenstates of the input field are uncorrelated. In other words, one may superpose two different inputs to the system either coherently or, as in this particular case, incoherently. However, we point out that, in general, the $S$ matrix operation does not preserve orthogonality. Thus, the output field vectors $\mathbf{e}_{j, 1, \pi \sigma}^{(m, n)}$ and $\mathbf{e}_{j, 2, \pi \sigma}^{(m, n)}$ for same $m, n$, and $j$, are not generally orthogonal. An exemplar of this kind of situation is the metal-stripe polarizer (see, for example, [18]): regardless of the state of polarization of the input field, the output field vectors are parallel. This property does not, however, affect the validity of Eq. (19).

Finally, let us summarize the general procedure with making use of the Stokes representation:

1. The input field, characterized by the parameters $\left(\theta_{\text {in }}, \phi_{\text {in }}, P_{\text {in }}, \vartheta_{\text {in }}, \varphi_{\text {in }}\right)$ is converted into a polarization matrix $\mathbf{J}_{\mathrm{in}, \sigma \pi}$ by using Eqs. (4)-(7).

2. The eigenvalues and eigenvectors of the input-field polarization matrix are solved by standard tools.

3. The fields characterized by the eigenvectors are propagated separately through the grating, which yields us the vectors $\mathbf{e}_{j, 1, \pi \sigma}^{(m, n)}$ and $\mathbf{e}_{j, 2, \pi \sigma^{\prime}}^{(m, n)} j=(\mathrm{r}, \mathrm{t})$, of the diffraction orders.

4. Polarization matrices of the orders are obtained from Eq. (19).

5. The parameters $\left[\theta_{j}^{(m, n)}, \phi_{j}^{(m, n)}, P_{j}^{(m, n)}, \vartheta_{j}^{(m, n)}, \varphi_{j}^{(m, n)}\right]$ for diffraction orders are obtained from Eqs. (4)-(7).

\section{POLARIZATION ANALYSIS WITH FOURIER MODAL METHOD}

Let us next turn to examine how we can employ the method discussed in preceding Section in the case of standard FMM [4] in the case that the the grating vectors are perpendicular to each other, i.e. the angle $\zeta$ in Ref. [4] is zero. In such a case, the internal coordinate system of the grating is the Cartesian one. Assuming that the input field is characterized by parameters $\left(\theta_{\text {in }}, \phi_{\text {in }}, P_{\text {in }}, \vartheta_{\text {in }}, \varphi_{\text {in }}\right)$, and that its amplitude is normalized to unity (and hence $S_{0, \text { in }}=1$ ), as is common practice within the grating theory, we first need to find the polarization matrix of the field. Consulting Eqs. we obtain the 
elements of the polarization matrix in the forms

$$
\begin{aligned}
J_{\text {in }, \pi \pi} & =\frac{1}{2}\left(1+P \sin \vartheta_{\text {in }} \cos \varphi_{\text {in }}\right) \\
J_{\text {in }, \pi \sigma} & =\frac{P}{2}\left(\sin \vartheta_{\text {in }} \sin \varphi_{\text {in }}+\mathrm{i} \cos \vartheta_{\text {in }}\right) \\
J_{\text {in }, \sigma \pi} & =\frac{P}{2}\left(\sin \vartheta_{\text {in }} \sin \varphi_{\text {in }}-\mathrm{i} \cos \vartheta_{\text {in }}\right) \\
J_{\text {in }, \sigma \sigma} & =\frac{1}{2}\left(1-P \sin \vartheta_{\text {in }} \cos \varphi_{\text {in }}\right) .
\end{aligned}
$$

We next solve the eigenvalues $I_{\text {in, } 1}$ and $I_{\text {in,2 }}$, as well as the eigenvectors $\mathbf{e}_{\mathrm{in}, 1, \pi \sigma}^{*}$ and $\mathbf{e}_{\mathrm{in}, 2, \pi \sigma}^{*}$ by any suitable way.

The complex conjugates of the eigenvectors can now be used as the inputs to the FMM code. In order to do that, let us first convert the vectors from $\pi \sigma$ system to the Cartesian basis, by using Eqs. (2), which yields

$$
\begin{aligned}
& e_{\mathrm{in}, \mathrm{p}, \mathrm{x}}=e_{\mathrm{in}, p, \pi} \cos \theta_{\mathrm{in}} \cos \phi_{\mathrm{in}}-e_{\mathrm{in}, p, \sigma} \sin \phi_{\mathrm{in}} \\
& e_{\mathrm{in}, p, y}=e_{\mathrm{in}, p, \pi} \cos \theta_{\mathrm{in}} \sin \phi_{\mathrm{in}}-e_{\mathrm{in}, p, \sigma} \cos \phi_{\mathrm{in}}
\end{aligned}
$$

where $p=(1,2)$. These vectors are now used separately as inputs to the system $S$-matrix, which yields us the complex amplitudes of the diffraction orders, typically in the form of $x, y$, and $z$, components of the electric field vectors. Note that one should solve the $S$-matrix only once, as it does not depend on the polarization state of the input. Again using Eqs. (2), we find the vector complex amplitudes $\mathbf{e}_{j, p, \pi \sigma}^{(m, n)}$, where $j=(\mathbf{r}, \mathrm{t})$ and $p=(1,2)$. The polarization matrices of the orders are now simply

$$
\mathbf{J}_{j, \pi \sigma}^{(m, n)}=I_{\mathrm{in}, 1}\left[\mathbf{e}_{j, 1, \pi \sigma}^{(m, n)}\right]^{*}\left[\mathbf{e}_{j, 1, \pi \sigma}^{(m, n)}\right]^{\mathrm{T}}+I_{\mathrm{in}, 2}\left[\mathbf{e}_{j, 2, \pi \sigma}^{(m, n)}\right]^{*}\left[\mathbf{e}_{j, 2, \pi \sigma}^{(m, n)}\right]^{\mathrm{T}},
$$

where $j=(\mathrm{r}, \mathrm{t})$. The degrees of polarization of the orders can be now found from $[13,14]$

$$
P_{j}^{(m, n)}=\left\{1-\frac{4 \operatorname{det} \mathbf{J}_{j, \pi \sigma}^{(m, n)}}{\left[\operatorname{tr} \mathbf{J}_{j, \pi \sigma}^{(m, n)}\right]^{2}}\right\}^{1 / 2}, j=(\mathrm{r}, \mathrm{t}),
$$

where det stands for the determinant. Moreover, the polarization angles are found using Eqs. (4) and (7), which yields

$$
\begin{aligned}
& \vartheta_{j}^{(m, n)}=\arccos \left\{\frac{2 \Im\left[J_{j, \pi \sigma}^{(m, n)}\right]}{P_{j}^{(m, n)} \operatorname{tr} \mathbf{J}_{j, \pi \sigma}^{(m, n)}}\right\}, \\
& \varphi_{j}^{(m, n)}=\arctan \left\{\frac{2 \Re\left[J_{j, \pi \sigma}^{(m, n)}\right]}{J_{j, \pi \pi}^{(m, n)}-J_{j, \sigma \sigma}^{(m, n)}}\right\}, \quad j=(\mathrm{r}, \mathrm{t}) .
\end{aligned}
$$

The diffraction efficiencies are obtained directly from Eq. (6).

\section{EXAMPLES}

Finally, let us study several examples on how polarization states of diffraction orders are changed upon propagation through one- or two-dimensionally modulated gratings. Assume first a simple two-level surface-relief linear grating, made into glass $(n=1.5)$. The period of the grating is $3.6 \lambda$, where $\lambda$ is the wavelength in vacuum, the thickness of the grating layer is $2.8 \lambda$, and the filling factor (linewidth-toperiod ratio) is 0.5 . We assume incidence from air, and that the field is characterized by parameters $\left(\theta_{\text {in }}, \phi_{\text {in }}, P_{\text {in }}, \vartheta_{\text {in }}, \varphi_{\text {in }}\right)=$ $\left(12^{\circ}, 90^{\circ}, 0,0,0\right)$, where the last two parameters are in fact free because the field is unpolarized. Thus, we are dealing the socalled pure conical mounting, the grating lines being parallel to the plane of incidence. Owing to the mirror-symmetric problem, it is expected that the efficiencies and degrees of polarization of diffraction orders are symmetric with respect to the zeroth order. Examining the results for the transmitted field, summarized in Table 1 and Figure 3, we find that this is indeed the case. Moreover, we find that majority of the orders has non-negligible degree of polarization, even though it remains quite low for first diffraction orders which contain the majority of the energy. It is remarkable that $\vartheta_{\mathrm{t}}^{(m)} \approx 90^{\circ}$ for every order, i.e. the orders may be called partially linearly polarized.

\begin{tabular}{r|rccc}
$m$ & $\eta_{\mathrm{t}}^{(m)}[\%]$ & $P_{\mathrm{t}}^{(m)}$ & $\vartheta_{\mathrm{t}}^{(m)}$ & $\varphi_{\mathrm{t}}^{(m)}$ \\
\hline \hline-5 & 0.9 & 0.35 & $93^{\circ}$ & $204^{\circ}$ \\
-4 & 3.9 & 0.03 & $94^{\circ}$ & $231^{\circ}$ \\
-3 & 2.4 & 0.62 & $88^{\circ}$ & $36^{\circ}$ \\
-2 & 4.5 & 0.26 & $86^{\circ}$ & $48^{\circ}$ \\
-1 & 32.1 & 0.10 & $94^{\circ}$ & $257^{\circ}$ \\
0 & 9.7 & 0.15 & $90^{\circ}$ & $180^{\circ}$ \\
+1 & 32.1 & 0.10 & $86^{\circ}$ & $103^{\circ}$ \\
+2 & 4.5 & 0.26 & $94^{\circ}$ & $312^{\circ}$ \\
+3 & 2.4 & 0.62 & $92^{\circ}$ & $324^{\circ}$ \\
+4 & 3.9 & 0.03 & $86^{\circ}$ & $129^{\circ}$ \\
+5 & 0.9 & 0.35 & $87^{\circ}$ & $156^{\circ}$ \\
\hline \hline
\end{tabular}

TABLE 1 Transmitted-orders efficiencies and polarization parameters for the first grating.

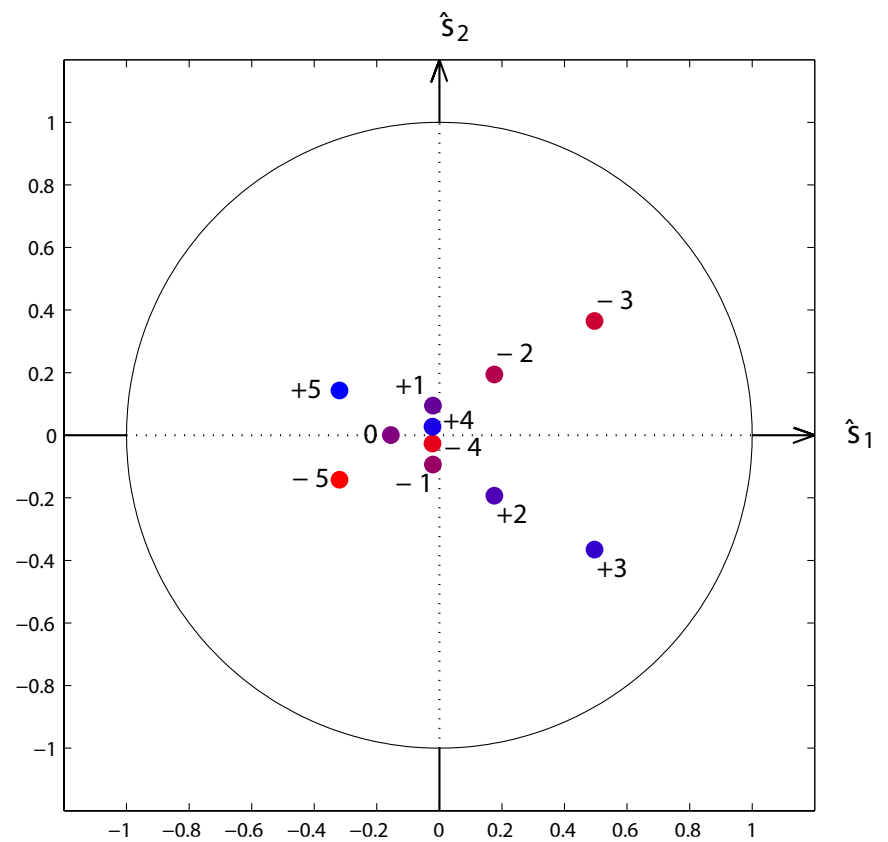

FIG. 3 Polarization states of transmitted orders in Poincare's sphere for the first grating. only planar projection is shown for clarity, since $s_{3}$ is very small for all orders.

We next consider the same grating, but this time illuminated by partially polarized input, characterized by param- 
eters $\left(\theta_{\text {in }}, \phi_{\text {in }}, P_{\text {in }}, \vartheta_{\text {in }}, \varphi_{\text {in }}\right)=\left(12^{\circ}, 90^{\circ}, 0.5,90^{\circ}, 180^{\circ}\right)$. The results, summarized in Table 2 and Figure 4 , show some interesting properties of the degrees of polarizations of the orders. In particular, some orders are less polarized than the input field, i.e. one may speak about depolarization of light. This property may sound somewhat astonishing, as the element is very thin deterministic optical element, but can be explained if we recall that the polarized part of the input field has in general different diffraction efficiencies than the unpolarized part.

\begin{tabular}{r|rcrr}
$m$ & $\eta_{\mathrm{t}}^{(m)}[\%]$ & $P_{\mathrm{t}}^{(m)}$ & $\vartheta_{\mathrm{t}}^{(m)}$ & $\varphi_{\mathrm{t}}^{(m)}$ \\
\hline \hline-5 & 0.7 & 0.19 & $80^{\circ}$ & $39^{\circ}$ \\
-4 & 3.9 & 0.48 & $80^{\circ}$ & $28^{\circ}$ \\
-3 & 3.1 & 0.85 & $88^{\circ}$ & $36^{\circ}$ \\
-2 & 5.1 & 0.67 & $89^{\circ}$ & $46^{\circ}$ \\
-1 & 30.5 & 0.42 & $90^{\circ}$ & $75^{\circ}$ \\
0 & 10.4 & 0.61 & $90^{\circ}$ & $180^{\circ}$ \\
+1 & 30.5 & 0.42 & $90^{\circ}$ & $285^{\circ}$ \\
+2 & 5.1 & 0.67 & $91^{\circ}$ & $314^{\circ}$ \\
+3 & 3.1 & 0.85 & $92^{\circ}$ & $324^{\circ}$ \\
+4 & 3.9 & 0.48 & $100^{\circ}$ & $332^{\circ}$ \\
+5 & 0.7 & 0.19 & $100^{\circ}$ & $321^{\circ}$ \\
\hline \hline
\end{tabular}

TABLE 2 Transmitted-orders efficiencies and polarization parameters for the second grating.

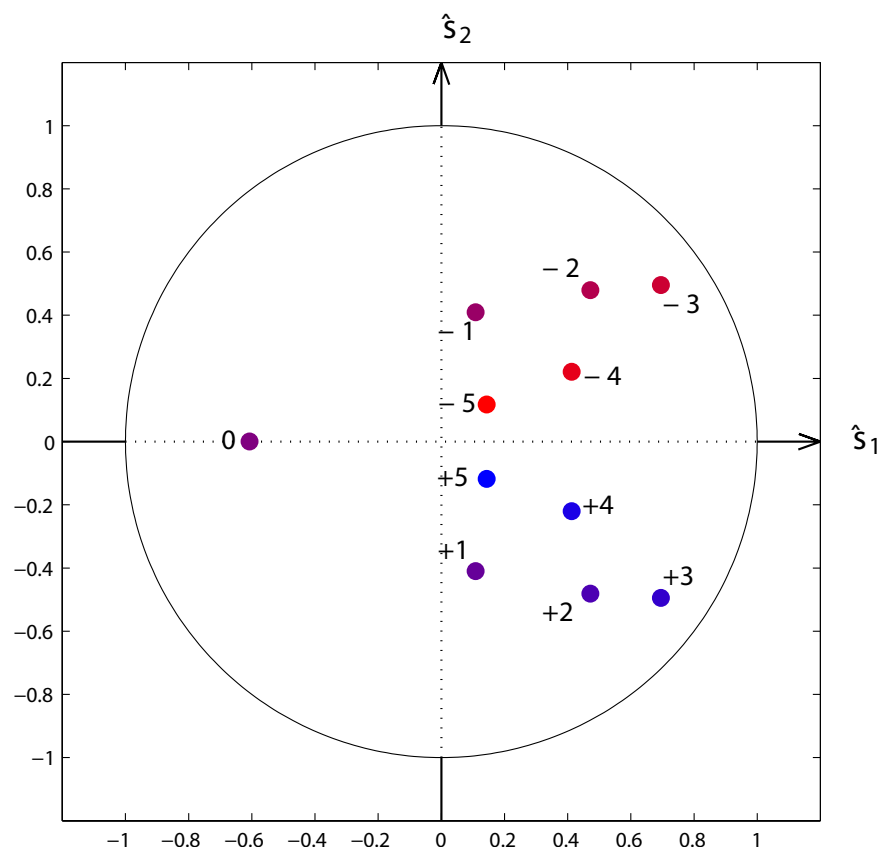

FIG. 4 Polarization states of transmitted orders in Poincare's sphere for the second grating. Again, only planar projection is shown for clarity, since $s_{3}$ is quite small for all orders.

Our third example deals with the so-called planar chiral metamaterials that attract much interest in recent years [19]-[23]. Since this kind of artificial nanostructures can produce large optical activity that originates from the structural chiral features, but not the intrinsic property of the materials, they are very promising to be developed as new generation polarization elements. Nowadays, the analysis of polarization conversion effect of the chiral nanograting is needed to be made for not only the directly transmitted order[21, 22], but also the higher diffracted orders $[19,20]$. However, if taking into account simultaneously the structural chirality, the arbitrary polarization state of input field, as well as the general diffraction configuration, one would realize that the theoretical analysis of the problem seems extremely complex if only applying the traditional rigorous diffraction theory of gratings; it is almost impossible to figure out the polarization states of all diffraction orders within one calculation. Nevertheless, with the approach the we proposed, the numerical analysis of the planar chiral metamaterials would be largely simplified.

We analyze as an example a gammadion-shaped nanoparticle array shown in Figure 5, where the structural parameters are also given. The spatial mounting and polarization state of the input field is characterized by $\left(\theta_{\text {in }}, \phi_{\text {in }}, P_{\text {in }}, \vartheta_{\text {in }}, \varphi_{\text {in }}\right)=$ $\left(10^{\circ}, 0^{\circ}, 0.5,60^{\circ}, 0^{\circ}\right)$, i.e., it is an oblique incident, partially elliptically polarized plane wave. As can be seen from the simulation results demonstrated in Table 3, the polarization states of all the transmitted orders are quite complex and are totally different from each other, which shows that the structural chirality imposes a strong effect on the polarization response in diffraction. With the previous numerical methods, the determination of the polarization state of each order is quite troublesome; however, with our approach, all the results were obtained at once without a perceivable increase of the computation time. So it is very hopeful that this approach can be used as a convenient tool in analyzing the polarization effect of planar chiral metamaterials.

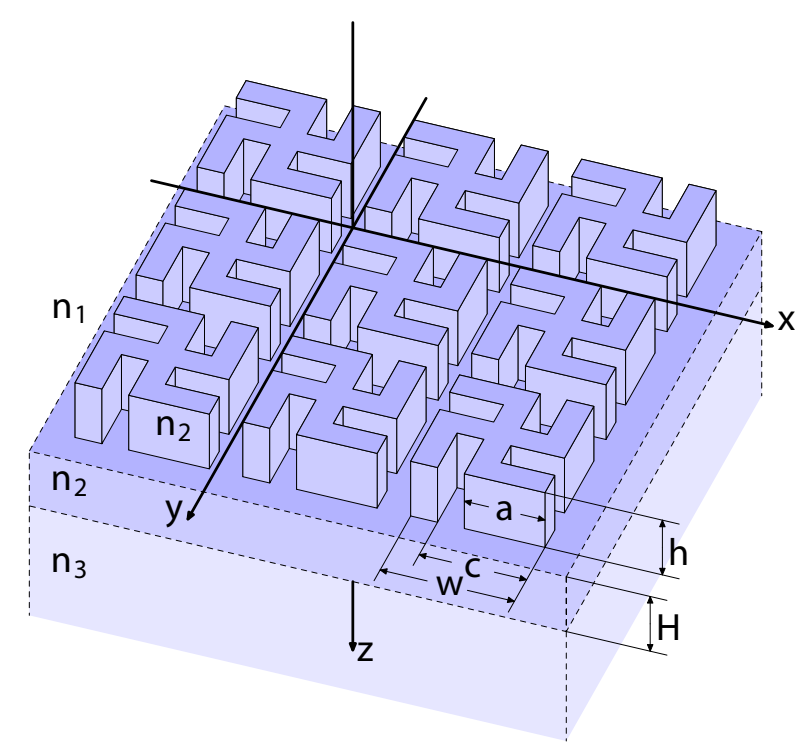

FIG. 5 Gammadion structure assumed in the third example. The grating parameters are $a=240 \mathrm{~nm}, c=320 \mathrm{~nm}, w=400 \mathrm{~nm}, h=H=200 \mathrm{~nm}, n_{1}=1, n_{2}=3$, and $n_{3}=1.5$. The grating parameters in the $y$-direction are the same as in the $x$-direction, and the grating period is $500 \mathrm{~nm}$. 


\begin{tabular}{r|rrrr}
$(m, n)$ & $\eta_{\mathrm{t}}^{(m, n)}[\%]$ & $P_{\mathrm{t}}^{(m, n)}$ & $\vartheta_{\mathrm{t}}^{(m, n)}$ & $\varphi_{\mathrm{t}}^{(m, n)}$ \\
\hline \hline$(0,-1)$ & 12 & 0.89 & $82^{\circ}$ & $132^{\circ}$ \\
$(-1,0)$ & 28 & 0.66 & $72^{\circ}$ & $49^{\circ}$ \\
$(0,0)$ & 24 & 0.30 & $108^{\circ}$ & $30^{\circ}$ \\
$(+1,0)$ & 5 & 0.62 & $92^{\circ}$ & $177^{\circ}$ \\
$(0,+1)$ & 16 & 0.92 & $77^{\circ}$ & $133^{\circ}$ \\
\hline \hline
\end{tabular}

TABLE 3 Transmitted-orders efficiencies and polarization parameters for the third grating.

\section{SUMMARY}

We have put forward a general approach for convenient analysis of diffraction problem of gratings under illumination of arbitrarily polarized light, which can be easily incorporated with the existing rigorous numerical methods of gratings. We also discussed how one should illustrate the polarization states of the input field and diffraction orders such that the results can be easily interpreted. The approach is based on wellknown properties of partially polarized radiation, in particular Stokes representation and polarization matrix approach, as well as rigorous diffraction theory of gratings. In addition, we have provided detailed guidelines of the implementation of the method, and illustrated the usefulness of the taken approach by numerical examples. It may be expected that the method will found its way in the analysis and design of polarization effects in diffraction gratings.

\section{ACKN OWLEDGMENTS}

The work was supported by the Academy of Finland (projects 111701 and 118951). The authors also acknowledge the Network of Excellence on Micro-Optics (NEMO) as well as The Research and Development Project on Nanophotonics (Ministry of Education, Finland).

\section{References}

[1] R. Petit (ed.), Electromagnetic Theory of Gratings (Springer, Berlin, 1980).

[2] E. Noponen and J. Turunen, "Eigenmode method for electromagnetic synthesis of diffractive elements with three-dimensional profiles" J. Opt. Soc. Am. A 11, 2494-2502 (1994).

[3] S. Peng and G. M. Morris, "Efficient implementation of rigorous coupled-wave analysis for surface-relief gratings" J. Opt. Soc. Am. A 12, 1087-1096 (1995).

[4] L. Li, "New formulation of the Fourier modal method for crossed surface-relief gratings" J. Opt. Soc. Am. A 14, 2758-2767 (1997).

[5] G. Granet, "Analysis of diffraction by surface-relief crossed gratings with use of the Chandezon method: application to multilayer crossed gratings" J. Opt. Soc. Am. A 15, 1121-1131 (1998).
[6] L. Li, "Fourier modal method for crossed anisotropic gratings with arbitrary permittivity and permeability tensors" J. Opt. A: Pure Appl. 0p. 5, 345-355 (2003).

[7] S. Siitonen, P. Laakkonen, P. Vahimaa, K. Jefimovs, M. Kuittinen, M. Parikka, K. Mönkkönen, and A. Orpana, "Coupling of light from an LED into a thin light guide by diffractive gratings" Appl. Optics 43, 5631-5635 (2004).

[8] S. Siitonen, P. Laakkonen, P. Vahimaa, M. Kuittinen, and N. Tossavainen, "White LED light coupling into light guides with diffraction gratings" Appl. Optics 45, 2623-2630 (2006).

[9] S. R. Park, O. J. Kwon, D. Shin, S.-H. Song, H.-S. Lee, and H. Y. Choi, "Grating micro-dot patterned light guide plates for LED backlights" Opt. Express 15, 2888-2899 (2007).

[10] S. Siitonen, P. Laakkonen, J. Tervo, and M. Kuittinen, "A doublesided grating coupler for thin light guides" Opt. Express 15, 20082018 (2007).

[11] L. Li, "Formulation and comparison of two recursive matrix algorithms for modeling layered diffraction gratings" J. Opt. Soc. Am. A 13, 1024-1035 (1996).

[12] M. Born and E. Wolf, Principles of Optics 7 th edn. (Cambridge University Press, Cambridge, UK, 1999).

[13] L. Mandel and E. Wolf, Optical Coherence and Quantum Optics (Cambridge University Press, Cambridge, UK, 1995).

[14] C. Brosseau, Fundamentals of Polarized Light: A Statictical Optics Approach (Wiley, New York, 1998).

[15] J. Tervo, T. Setälä, and A. T. Friberg, "Theory of partially coherent electromagnetic fields in the space-frequency domain" J. Opt. Soc. Am. A 21, 2205-2215 (2004).

[16] J. Tervo and J. Turunen, "Angular spectrum representation of partially coherent electromagnetic fields" Opt. Commun. 209, 7-16 (2002).

[17] L. Mandel, "Intensity fluctuations of partially polarized light" Proc. Phys. Soc. 81, 1104-1114 (1963).

[18] M. Honkanen, V. Kettunen, M. Kuittinen, J. Lautanen, J. Turunen, B. Schnabel, and F. Wyrowski, "Inverse metal-stripe polarizers" Appl. Phys. B - Lasers 0. 68, 81-85 (1999).

[19] A. Papakostas, A. Potts, D. M. Bagnall, S. L. Prosvirnin, H. J. Coles, and N. I. Zheludev, "Optical manifestations of planar chirality" Phys. Rev. Lett. 90, 107404 (2003).

[20] W. Zhang, A. Potts, and D. M. Bagnall, "Giant optical activity in dielectric planar metamaterials with two-dimensional chirality" J. Opt. A: Pure Appl. Op. 8, 878-890 (2006).

[21] M. Kuwata-Gonokami, N. Saito, Y. Ino, M. Kauranen, K. Jefimovs, T. Vallius, J. Turunen, and Y. Svirko, "Giant optical activity in quasi-two-dimensional planar nanostructures" Phys. Rev. Lett. 95, 227401 (2005).

[22] B. Bai, Y. Svirko, J. Turunen, and T. Vallius, "Optical activity in planar chiral metamaterials: Theoretical study" Phys. Rev. A 76, 023811 (2007).

[23] M. Decker, M. W. Klein, M. Wegener, and S. Linden, "Circular dichroism of planar chiral magnetic metamaterials" Opt. Lett. 32, 856-858 (2007). 